\title{
New Dimensions for Adolescent Substance Use Prevention
}

\author{
Susan R. Tortolero
}

Published online: 25 November 2012

(C) Springer Science+Business Media New York 2012

The primary focus of this issue of The Journal of Primary Prevention (JPP) - adolescent substance use-is particularly important to me. As a researcher in adolescent health and the parent of a teenager, I continually monitor the challenges adolescents face in the complex world we live in and examine the most effective ways to prepare them to meet those challenges. Thus, I benefit greatly when research converges to address health and behavioral outcomes that impact me not only professionally (as a researcher) but also personally (as a parent). I trust that $J P P$ readers will feel similarly.

Adolescent substance use continues to be a major public health problem in the United States. While tobacco and alcohol use have declined among U.S. teens over the past 20 years, marijuana use has increased. According to the Youth Risk Behavior Surveillance Survey (Centers for Disease Control and Prevention 2012), past-month alcohol use decreased by $24 \%$, binge drinking by $24 \%$, and past-month cigarette use by $34 \%$ among U.S. high school students from 1991 to 2011. However, during this same time period, marijuana use increased by $60 \%$, with $23 \%$ of U.S. high school students reporting having smoked marijuana in the past 30 days. It is important to emphasize that adolescent substance use is not only a national public health problem but also an international

S. R. Tortolero $(\square)$

University of Texas School of Public Health,

Houston, TX, USA

e-mail: Susan.Tortolero@uth.tmc.edu one, impacting teens in various communities around the world.

Two studies in this issue examined the potential impact of religiosity and spirituality on adolescent substance use. Both of these studies are intriguing given growing evidence suggesting that religiosity and spirituality are protective against substance use, especially among urban adolescents. In a sample of predominantly African American youth, aged 13-20 years and living in Philadelphia, Mason et al. tested whether three dimensions of religiosity (social religiosity, perceived religious support, and private religiosity) as well as proximity to religious institutions were protective against various types of substance use. Contributing to the novelty of this study is the fact that the authors investigated the impact of multiple dimensions of religiosity, which in adolescent substance use research has typically been assessed using a single dimension. Logistic regression analysis revealed that two dimensions of religiosity were protective against at least one type of substance use: Social religiosity was protective against marijuana use, and perceived religious support was protective against tobacco use. However, the third dimension of religiosity, private religiosity, was not protective against any type of substance use. As Mason et al. conclude, in addition to providing initial evidence that proximity to religious institutions is protective against alcohol use, their findings point to the importance of examining religiosity as a multidimensional construct when investigating substance use in urban youth populations. 
In their study, Pokhrel et al. tested whether spirituality and heath-as-a-value had protective effects on recent cigarette and alcohol use behaviors and future cigarette and alcohol use intentions among Russian adolescents living in Ufa, the capital city of the Republic of Bashkortostan in Russia. This study extends research on these two constructs beyond the borders of the United States. Additionally, this study is important not only because it informs the growing body of adolescent substance use research by providing an international perspective but also because, according to the national estimates cited by the authors, the prevalence of tobacco and alcohol use is higher among Russian adolescents than among U.S. adolescents. In a sample of Russian high school students, aged 14-18 years, Pokhrel et al. found a significant inverse relationship between health-as-avalue and past-month cigarette and alcohol use as well as next-year cigarette use intentions. However, they did not find a significant relationship between spirituality and any of the substance use variables included in their analysis. This null finding is particularly intriguing given that research conducted in the United States has generally shown spirituality to be protective against substance use. In addition to indicating that spirituality may not be an important component of personal identity among Russian adolescents as among their American counterparts, as the authors conclude, the null finding suggests that spirituality may not be universally protective against substance use in urban youth populations, having a differential impact across cultures.

Two more studies in this issue focused on adolescent substance use but are methodological in nature. In their study, Briney et al. utilized longitudinal data to determine the predictive validity of the cut-point values for scales measuring risk and protective factors on the Communities That Care Youth Survey (CTCYS), which were previously established using cross-sectional data. The CTCYS measures 32 risk and protective factors as well as 22 behavioral outcomes such as drug use and delinquent behaviors. Data were drawn from a panel of elementary school students living in 12 U.S. communities, who were assessed for substance use and delinquent behavior in middle school. Overall, Briney et al.'s findings indicate the validity of established cut-point values for risk and protective scales in predicting future drug use and delinquent behaviors among youth. Thus, as the authors conclude, these cut points can be used to identify elevated risk factors and depressed protective factors in a community, enabling the planning and tailoring of prevention activities as needed.

Lastly, in a "Research Methods and Practice" article, Okamoto et al. describe the early developmental stages of a drug prevention program for rural Hawaiian youth. Specifically, the authors describe the adaptation and validation of narrative scripts focused on offers of various substances from various individuals, which will be used to create culturally grounded videos. These videos will ultimately be incorporated into a school-based drug prevention curriculum for rural Hawaiian youth, who have been the focus of limited prevention research despite being characterized by disproportionate substance use rates. Middle school- and high school-aged youth across 15 focus groups adapted and validated these scripts to make them "more realistic" in the context of rural Hawai ' $i$. Through the adaptation and validation processes, youth suggested changes to the setting and behaviors depicted in the scripts. Okamoto et al. also observed that youth's preference for a particular type of drug resistance strategy (indirect vs. direct) was largely contingent on the identity of the drug offerer. As the authors conclude, their findings have implications for the ongoing development of this video-enhanced, culturally grounded drug prevention curriculum.

By any measure, adolescent substance use is unacceptably high and has major consequences for the health of adolescents, not only those living in the United States but also those living in other countries. It is clear that we still have far to go in understanding determinants of youth risk behaviors, in developing measures to study these behaviors and their determinants, and in developing intervention strategies that are culturally relevant for diverse adolescent populations. This issue of JPP makes an important contribution to that growing body of knowledge.

\section{Reference}

Centers for Disease Control and Prevention. (2012, August). Youth Risk Behavior Surveillance System (YRBSS). Retrieved from http://www.cdc.gov/yrbss. 